\title{
O USO DA INTEGRAL DEFINIDA NO CÁLCULO DA ÁREA ALAGADA DA BARRAGEM DO RIO BONITO
}

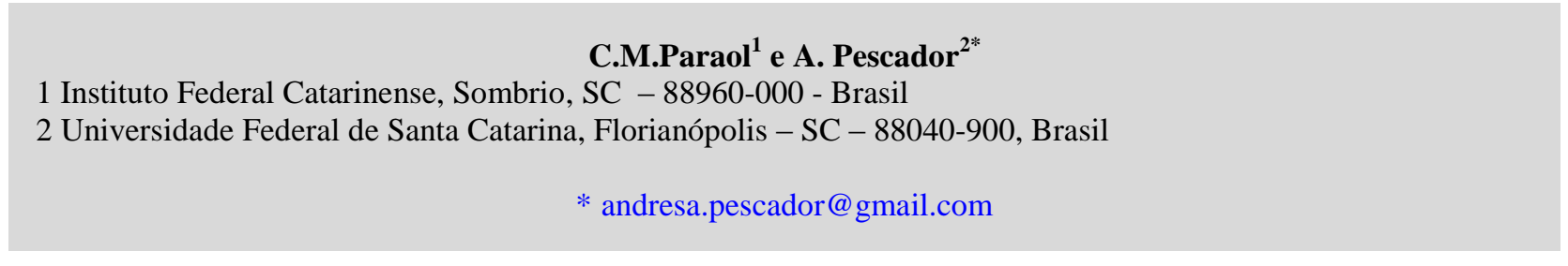

Artigo submetido em 27/08/2015 e aceito em 28/12/2015

\section{RESUMO}

Este artigo apresenta uma aplicação de integral definida cujo objetivo é calcular a área alagada da barragem do rio Bonito. Os registros históricos mostram a importância desta barragem para a região, a mesma é responsável por conter as cheias no "Banhado do Sombrio", e pela irrigação das lavouras de arroz. O formato da barragem está longe de ser uma região regular, cujo cálculo da área seria facilmente encontrado. Para tanto, fez-se o estudo detalhado sobre aproximação para integrais definidas e ajustes de curvas. Estes temas foram usados na aproximação da área alagada da barragem do Rio Bonito. Para facilitar os cálculos, fez-se o uso dos softwares geogebra e graph.

PALAVRAS-CHAVE: Integral definida; Ajuste de curvas; geogebra e graph.

\section{THE USE OF INTEGRAL DEFINED TO CALCULATE THE AREA FLOODED OF RIO BONITO'S DAM}

\begin{abstract}
This paper presents an application of definite integral whose objective is to calculate the flooded area of Rio Bonito's dam. Historical records show the importance of this dam to the region, it is responsible for contain the floods in "Banhado do Sombrio ", and the irrigation of rice fields. The dam format is far from a regular region
\end{abstract}

whose area calculation would be easily found. We study definite integrals and curve fitting. These themes were used to aproximate the flooded area of Rio Bonito's dam. To facilitate the calculations, we use of GeoGebra and graph softwares.

KEYWORDS: integral defined; curve fitting; geogebra and graph. 


\section{INTRODUÇÃO}

Aplicações dos conteúdos de cálculo diferencial e integral são elementos de motivação para os cursos de matemática, física, engenharias e áreas afins. Segundo Kaiber e Renz (2008), no Brasil o ensino do cálculo diferencial e integral, historicamente, caracteriza-se pela prevalência de processos algébricos, seguidos de exercícios repetitivos e com pouca, ou quase nenhuma interdisciplinaridade. No entanto, diversos estudos têm sido feitos, a fim de testar e qualificar metodologias para o ensino do cálculo.

De acordo com Stewart (2011), uma das aplicações da integral definida é o cálculo de área, onde a área é dividida em retângulos e a área exata é o limite das somas desses retângulos.

Com o intuito de apresentar uma aplicação de integrais, o objetivo deste artigo consiste em calcular a área alagada pela barragem do rio Bonito, que apresenta um formato irregular, utilizando os conceitos de integral definida e ajuste de curvas.

A barragem está localizada na comunidade de Tenente, no município de Jacinto Machado SC. A construção das barragens do rio Leão e do rio Bonito, fazem parte do "Projeto de Desenvolvimento Agrícola do Banhado de Sombrio". A represa do rio Bonito, objeto de estudo deste artigo foi concluída no ano de 1994, e neste mesmo ano foi fundada a COOIJAM (Cooperativa de Irrigação de Jacinto Machado). A cooperativa é responsável pela administração e fiscalização das águas da barragem, e entre os seus principais objetivos estão: conter as cheias e irrigar as lavouras de arroz.

Para facilitar o desenvolvimento dos cálculos, foram utilizados os softwares geogebra $4.4 \mathrm{e}$ graph 4.3. Segundo Kaiber e Renz (2008), a utilização de tecnologias no ensino da matemática fazse necessária para que a educação cumpra seu papel de preparar o indivíduo para a vida social e para o mundo do trabalho. Utilizar softwares matemáticos, motiva os alunos, possibilitando-lhes, desenvolver a capacidade de interpretar, analisar e estabelecer conjecturas, favorecendo a construção sólida dos conhecimentos.

$\mathrm{O}$ artigo é organizado da seguinte forma. Na seção 1 apresenta-se a introdução com os objetivos e a problemática a ser resolvida. Na seção 2 os materiais e métodos. O desenvolvimento do trabalho apresenta-se na seção 3. E finalmente, na seção 4, apresentam-se as considerações finais deste artigo.

\section{MATERIAIS E MÉTODOS}

\subsection{Histórico da Barragem do Rio Bonito}

Na década de 70 a SUDESUL (Superintendência do Desenvolvimento da Região Sul) iniciou o desenvolvimento de um grande projeto, cujo interesse foi motivado pela presença do carvão coqueificável na região carbonífera. Ligada ao Ministério do Interior, a SUDESUL buscava a criação de um pólo econômico desenvolvendo aquele que chamou de Projeto Litoral Sul de Santa Catarina (PLSSC).

O Projeto Litoral Sul de Santa Catarina, fez nascer o "projeto de desenvolvimento agrícola do banhado de sombrio", que tinha por objetivo principal o desenvolvimento hortigranjeiro daquela região. O projeto foi estruturado inicialmente com a construção de três grandes obras: a Escola Agrotécnica Federal de Sombrio (EAFS) e as barragens do Rio Bonito (figura 1) e do Rio Leão. 


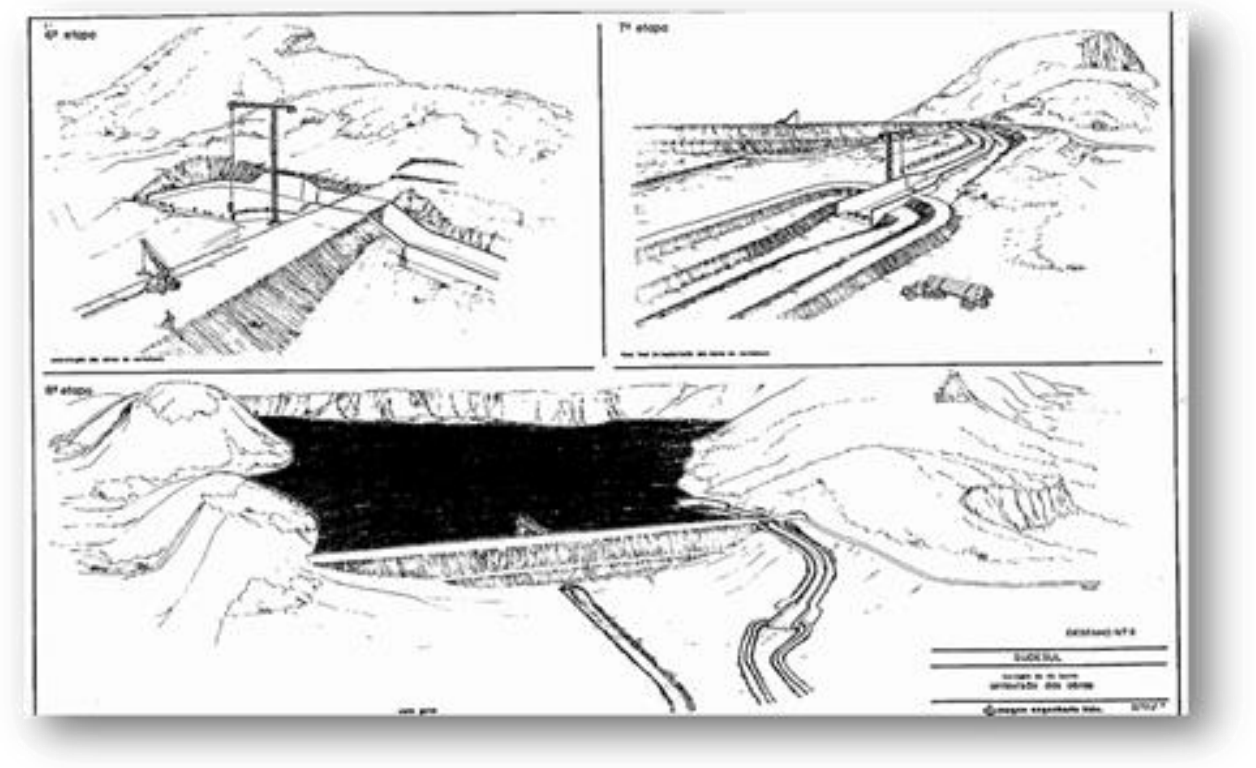

Figura 1 - Projeto da Barragem rio Bonito

Fonte: Magna Engenharia

A posição escolhida para a construção do barramento foi determinada pelo "estudo de viabilidade técnico-econômica do projeto de desenvolvimento agrícola do banhado de sombrio". Assim duas barragens distintas foram construídas na localidade de Tenente no município de Jacinto Machado, sul de Santa Catarina.

Os rios Leão e Bonito fazem parte da bacia do rio Mampituba, o qual é o limite entre os estados de Santa Catarina e Rio Grande do Sul. O rio Mampituba é o escoadouro natural das enchentes de seu vale, cujas chuvas nas encostas dos Aparados da Serra, descem pelos seus afluentes causando inundações e limitando o uso de solos férteis no alto, médio e baixo vale. $\mathrm{O}$ problema ocorre anualmente, então para solucionar tal problema a solução encontrada foi a construção de barramento em pontos estratégicos do vale.

A barragem do rio Bonito (figura 2), o qual é objeto de estudo deste artigo localiza-se no último estrangulamento deste rio, antes do mesmo ingressar na planície costeira, logo a montante na localidade de Tenente. Segundo informações do presidente da COOIJAM, a barragem tem aproximadamente 85 hectares de área alagada e foi concluída quatro anos antes da barragem do rio Leão. Os objetivos principais das barragens são: Acumular um volume suficiente de água, a fim de garantir uma vazão regularizada que permite irrigar as lavouras a jusante (lado para onde se dirige a corrente de água), pertencentes ao projeto de desenvolvimento agrícola do banhado de Sombrio; e laminar os hidrogramas de cheias afluentes ao reservatório, de forma que a vazão efluente possa ser conduzida pelos canais de macro drenagem ora em construção sem causar inundações. 


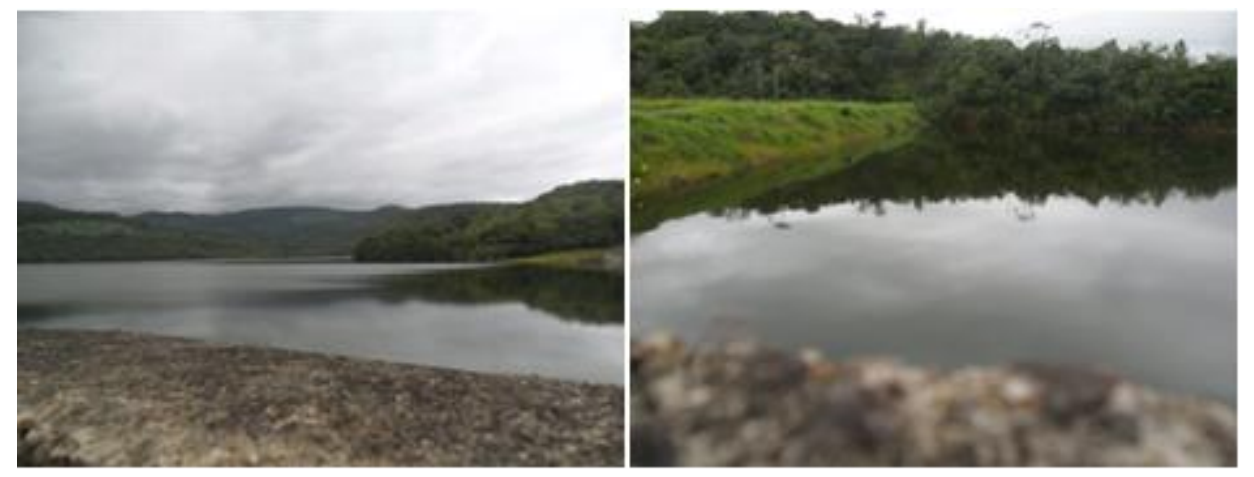

Figura 2 - Imagens da Barragem do rio Bonito Fonte: Autores, 2014.

Porém com todas as limitações físicas, burocráticas e humanas, o projeto se desenvolvia lentamente. No ano de 1990, cerca de 20 anos após o início do projeto, a nova gestão presidencial anunciou a extinção da SUDESUL, causando o abandono total das obras.

Após três anos de abandono, por intervenção da Unisul (Universidade do Sul de Santa Catarina) e da própria EAFS (Escola Agrotécnica Federal de Sombrio), o projeto foi retomado por parte dos governos estadual e federal. Em 1994 a barragem do rio Bonito estava concluída e alagada, neste mesmo ano no mês de outubro foi fundada a COOIJAM. Atualmente a cooperativa conta com 158 sócios, e dentre as suas múltiplas finalidades é responsável pela irrigação de 2910 hectares de arroz.

\subsection{Revisão Histórica do Cálculo Diferencial e Integral}

A história do cálculo diferencial e integral foi motivada a partir da necessidade de resolver dois problemas básicos: (i): O problema básico do cálculo diferencial, que é o problema das tangentes; ou seja, calcular o coeficiente angular da reta tangente ao gráfico de uma função num ponto dado P. (ii): O problema básico do cálculo integral, que é o problema das áreas; ou seja, calcular a área sob o gráfico, entre os pontos $x=a$ e $x=b$.

Há registros que os egípcios, babilônicos e gregos já utilizavam os conceitos de cálculo para resolver problemas envolvendo áreas e volumes.

O desenvolvimento do cálculo diferencial e integral teve a contribuição de vários matemáticos no decorrer da história. Porém, foi no século XVII, a partir dos trabalhos dos gregos Eudoxo (406-355 a.C.) e Arquimedes(287-212 a.C.) que os conceitos de integral começaram a se desenvolver.

Segundo Brandão et al (2007), a ideia básica do conceito de integral já estava embutida no método da exaustão atribuído a Eudoxo. O método da exaustão consiste em "exaurir" a figura dada por meio de outras figuras de áreas ou de volumes conhecidos.

Arquimedes foi quem aperfeiçoou o método de exaustão, o mesmo utilizou o método de exaustão para encontrar a área do círculo, e obteve as primeiras aproximações do número " $\pi$ ". Ainda, concluiu que a área da região limitada por uma parábola cortada por uma corda qualquer, é igual a 4/3 da área do triângulo que tem a mesma altura e que tem a corda como base.

Arquimedes é considerado um dos maiores matemáticos da antiguidade, e seu trabalho foi uma das maiores contribuições grega para o cálculo diferencial e integral. 
De acordo com Boyer (1994), Galileu Galilei (1564-1642) e Johann Kepler (1571-1630) estudaram os métodos de Arquimedes. Kepler utilizou os conceitos de integração, a fim de calcular as áreas envolvidas nas suas leis do movimento planetário. Kepler considerou a circunferência como um polígono regular de infinitos lados, onde cada um desses lados era à base de um triângulo e o vértice era o centro da circunferência. O círculo ficava dividido em uma infinidade de triângulos, todos com altura igual ao raio da circunferência. Como a área desses triângulos é dada pelo semiproduto da base pela altura, tem-se que a área do círculo é o semiproduto do comprimento da circunferência pelo raio. Kepler também obteve o volume da esfera e desenvolveu conhecimentos relacionados ao volume de diferentes sólidos de revolução.

Cavalieri (1598-1647) foi outro matemático que contribuiu para o desenvolvimento do cálculo diferencial e integral, o mesmo apresentou o método dos indivisíveis. O método considerava que a área era formada por segmentos indivisíveis, e que o volume pode ser considerado como composto de áreas que são volumes indivisíveis ou quase atômicos.

Outras contribuições significativas para o desenvolvimento do cálculo diferencial e integral vieram no século $X V I I$, que foi marcado por grandes avanços na área da ciência. Os matemáticos René Descartes (1596-1650) e Pierre Fermat (1601-1665) apresentaram as coordenadas cartesianas. De acordo com Pires et al (2008), as coordenadas cartesianas possibilitaram transformar e representar problemas geométricos em problemas algébricos e estudar funções analiticamente.

Segundo Boyer (1994), em relação ao matemático Fermat, uma de suas principais contribuições foi o desenvolvimento do método de encontrar máximos e mínimos. Fermat desenvolveu um método para encontrar máximos e mínimos de curvas polinomiais do tipo $y=f(x)$ desde que estes existam. O mesmo comparou o valor de $f(x)$ (imagem de $x$ pela função $f(x))$ com o valor de $f(x+\varepsilon)$, sendo $(x+\varepsilon)$ uma vizinhança de $x$, para todo $\varepsilon>0$ tão pequeno quanto se queira. Em geral, estas imagens são distintas e numa curva suave, sua variação é quase imperceptível. Os pontos máximos e mínimos eram determinados igualando $f(x)$ e $f(x+\varepsilon)$, assim Fermat percebeu que os valores eram quase todos iguais. Então quanto menor o valor de $\varepsilon$, mais próximo chega-se da igualdade, assim Fermat dividiu tudo por $\varepsilon$, e depois igualou $\varepsilon$ a zero. Este processo é equivalente ao de diferenciação usado nos dias atuais, ou seja, Fermat estava calculando exatamente o limite dado pela equação (1):

$$
\lim _{\varepsilon \rightarrow 0} \frac{f(x)-f(x+\varepsilon)}{\varepsilon}=0
$$

Em outras palavras, Fermat não conhecia o conceito de limite, porém seu método para encontrar máximos e mínimos é praticamente o mesmo utilizado hoje em dia. A diferença é que no lugar do $\varepsilon$, na maioria das vezes, usa-se o símbolo $h$ ou $\Delta x$. O processo utilizado por Fermat em mudar a variável e considerar os valores vizinhos é a essência da análise infinitesimal, ou seja, é exatamente o cálculo da derivada.

De acordo com Pires et al (2008), outra contribuição para o desenvolvimento do cálculo diferencial e integral foi do matemático Isaac Barrow (1630-1677), que aplicou com êxito a geometria e o cálculo diferencial e integral à óptica, definiu a derivada, porém, não havia uma fundamentação em seus trabalhos. Mais tarde esta fundamentação foi desenvolvida por Isaac Newton. 
O Cálculo Diferencial e Integral teve a contribuição de vários matemáticos no decorrer da história. Porém deve-se a Isaac Newton (1642-1727) e Wilhelm Leibniz (1646 -1716) a criação do teorema fundamental do cálculo. Segundo Stewart (2011), ambos descobriram a relação inversa existente entre a derivada e a integral, sistematizaram e aperfeiçoaram os conhecimentos produzidos na construção do cálculo diferencial e integral até então, e de forma independente e simultânea apresentaram o teorema fundamental do cálculo no final do século XVII.

De acordo com Boyer (1994), a primeira publicação das descobertas de Newton aconteceu no ano de 1687 no livro Philosophia e naturalis principia mathematica. Isaac Newton não foi o primeiro a diferenciar ou integrar, porém sua descoberta consistiu na sistematização de um algoritmo geral para funções.

No ano de 1684, Leibniz faz sua primeira publicação sobre o cálculo diferencial e integral, cujo título é Nova methodus pro maximis et minimis, item que tangentibus, quae nec fractas nec irrationales quantitates moratur, et singulare pro illis calculi genus (Um novo método para máximos e mínimos, e também para tangentes, que não é obstruído por quantidades irracionais). A notação e o nome "calculus differentialis e calculus integralis" (cálculo diferencial e integral) foi formalizada por Leibniz que fixou em $d x$ e $d y$ os diferenciais de $x$ e $y$.

O trabalho de Newton e Leibniz continuou sendo desenvolvido por outros matemáticos entre estes, os irmãos Bernoulli.

A definição da integral utilizada atualmente deve-se ao matemático francês Augustin-Lois Cauchy (1789-1857). Cauchy definiu que uma função $f$ é o limite de uma soma infinita. Após está definição ele demonstrou algumas propriedades, e concluiu que todas as funções contínuas em um intervalo $[a, b]$ são integráveis. O símbolo da integral, $\int$, é proveniente do (S) de soma "esticada", notação atribuída ao matemático Wilhelm Leibniz.

A definição da integral de Cauchy foi reformulada pelo matemático alemão Bernhard Riemann (1826-1866). Riemann definiu a Integral de uma função limitada num intervalo usando somas superiores e inferiores. Devido a está contribuição e em sua homenagem, a "integral de Riemann" é utilizada na disciplina de cálculo das universidades.

A teoria desenvolvida e as aplicações do Cálculo diferencial e integral estão entre as maiores realizações de todos os tempos. Kouropatov e Dreyfus (2009) ressaltam a importância da derivada, que junto com a integral Forman um núcleo de domínio matemático que é um dispositivo útil para todos os campos como: a física, a engenharia, a economia e a estatística. O conceito de integral representa uma ideia filosófica para a compreensão do mundo, onde a totalidade das partes pequenas de um todo remete a conclusões sobre o todo geral.

\subsection{Fundamentação Matemática}

De acordo com Santos et al (2012) é imprescindível a integração da Tecnologia e o Ensino de Matemática, porém o educador matemático deve estar preparado para compatibilizar os métodos de ensino e teorias de trabalho com as tecnologias. $O$ autor acrescenta que a utilização de recursos tecnológicos possibilitará uma visualização satisfatória dos conceitos matemáticos.

Para tanto, ao realizar este trabalho fez-se um estudo detalhado dos conteúdos de integral definida e ajuste de curvas, sendo que os cálculos para aproximação da área em questão foram desenvolvidos com o auxilio dos softwares geogebra e graph. O uso desta tecnologia facilitou consideravelmente o trabalho devido à quantidade de aproximações e divisões que foram feitas para o cálculo da área citada. 


\subsubsection{A Integral de Riemann}

Segundo Stewart (2011), a integral de Riemann é uma homenagem ao matemático Bernhard Riemann (1826-1866): “A integral definida de uma função integrável pode ser aproximada com qualquer grau de precisão desejado por uma soma de Riemann”.

Ainda segundo o autor, a integral definida por Riemann, consiste em dividir um intervalo $[a, b]$ em $n$ subintervalos de comprimentos iguais $\Delta x=\frac{b-a}{n}$, ou seja, dividir o intervalo em $n$ retângulos (figura 3). Para tal procedimento a função deve ser contínua e definida em $a \leq x \leq b$. Segue sua definição formal na equação (2).

$\int_{a}^{b} f(x) d x=\lim _{n \rightarrow \infty} \sum_{i=1}^{n} f\left(x_{i}^{*}\right) \Delta x$

onde $x_{0}(=a), x_{1}, x_{2}, \ldots, x_{n}(=b)$ são extremidades desses subintervalos, e $x_{1}^{*}, x_{2}^{*}, \ldots, x_{n}^{*}$ são pontos amostrais nestes subintervalos, de forma que $x_{i}^{*}$ esteja no i-ésimo subintervalo.

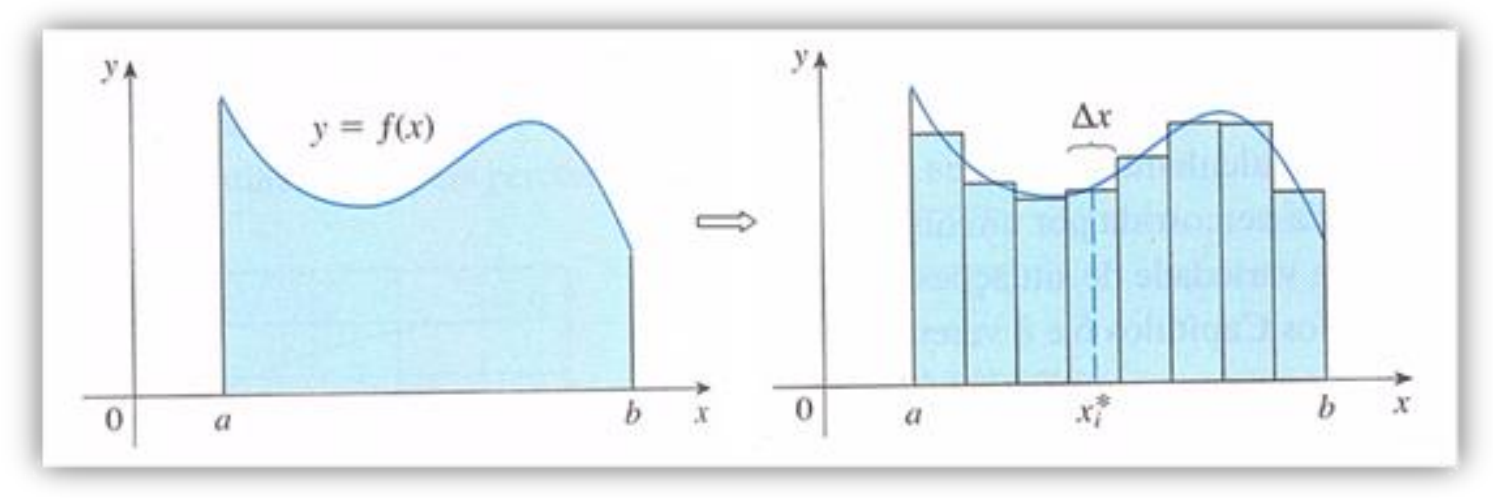

Figura 3 - Integral de Bernhard Riemann Fonte: Stewart, 2011, p. 346.

Nas aulas de geometria, aprende-se que área é um número que representa o tamanho de uma região limitada, e para regiões simples, como retângulos, triângulos, círculos, a área pode ser determinada por meio de fórmulas geométricas. Mas, no caso da área de regiões que não formam um padrão, ou seja, como no caso da (figura 3) se utiliza a integral definida para calcular a área de cada subintervalo, ou seja, a área da região sob a curva $f(x)$ no intervalo $[a, b]$ é aproximadamente a soma das áreas dos retângulos.

\subsubsection{Ajuste de curvas por Quadrados Mínimos}

Segundo Bassanezi (2011), o ajuste de curvas ou regressão é um artifício que expressa uma tendência entre a variável dependente versus a independente. O termo regressão surgiu no século $X I X$, quando Sir Francis Galton estudou a relação entre a altura de pais e filhos. O mesmo observou que havia um decréscimo na média, com relação aos valores encontrados entre as duas gerações. 
O método dos Quadrados Mínimos é uma das técnicas de aproximação mais usadas em problemas práticos. Isto se deve tanto à sua simplicidade quanto ao fato de que, em geral, buscamse aproximações para dados que são medidas obtidas experimentalmente com certo grau de incerteza. Nesta seção, apresenta-se o Método dos Quadrados Mínimos através do critério de minimizar os resíduos, para cada ponto.

Sendo que, mostra-se o caso particular do ajuste da função quadrática, que é aplicada na aproximação das funções a serem integradas para o cálculo da área. Em outras palavras, buscam-se pelos valores $a$, $b$ e $c$ que tornam a função $y_{i}=f\left(x_{i}\right)=a+b x_{i}+c x_{i}^{2}$ uma boa aproximação para os dados obtidos.

Assim, para encontrar a melhor aproximação para a curva, o critério adotado é minimizar a soma dos quadrados dos resíduos pontuais, isto é, minimizar a equação seguinte:

$$
\sum_{i=1}^{n} d^{2}\left(x_{i}\right)=\sum_{i=1}^{n}\left(y_{i}-\left(a+b x_{i}+c x_{i}^{2}\right)\right)^{2}
$$

Onde $y_{i}, i=1, \cdots, n$ são os dados que são aproximados. Este critério procura tornar os resíduos pontuais tão pequenos quanto possível. Para fazer a minimização da equação (3), segundo os conceitos do cálculo diferencial e integral, devem-se calcular as derivadas parciais da função objetivo em relação a cada uma das incógnitas, e devem-se igualar cada uma das derivadas parciais a zero:

$$
\begin{aligned}
& \frac{\partial D}{\partial a}=2 \sum_{i=1}^{n}\left(y_{i}-a-b x_{i}-c x_{i}^{2}\right) \cdot(-1)=0 \\
& \frac{\partial D}{\partial b}=2 \sum_{i=1}^{n}\left(y_{i}-a-b x_{i}-c x_{i}^{2}\right) \cdot\left(-x_{i}\right)=0 \\
& \frac{\partial D}{\partial c}=2 \sum_{i=1}^{n}\left(y_{i}-a-b x_{i}-c x_{i}^{2}\right) \cdot\left(-x_{i}^{2}\right)=0
\end{aligned}
$$

Assim, reescrevendo as igualdades acima, tem-se, respectivamente:

$$
\begin{aligned}
& -\sum_{i=1}^{n} y_{i}+\sum_{i=1}^{n} a+\sum_{i=1}^{n} b x_{i}+\sum_{i=1}^{n} c x_{i}^{2}=0 \Rightarrow a \sum_{i=1}^{n} 1+b \sum_{i=1}^{n} x_{i}+c \sum_{i=1}^{n} x_{i}^{2}=\sum_{i=1}^{n} y_{i} \\
& -\sum_{i=1}^{n} x_{i} y_{i}+\sum_{i=1}^{n} a x_{i}+\sum_{i=1}^{n} b x_{i}^{2}+\sum_{i=1}^{n} c x_{i}^{3}=0 \Rightarrow a \sum_{i=1}^{n} x_{i}+b \sum_{i=1}^{n} x_{i}^{2}+c \sum_{i=1}^{n} x_{i}^{3}=\sum_{i=1}^{n} x_{i} y_{i} \\
& -\sum_{i=1}^{n} x_{i}^{2} y_{i}+\sum_{i=1}^{n} a x_{i}^{2}+\sum_{i=1}^{n} b x_{i}^{3}+\sum_{i=1}^{n} c x_{i}^{4}=0 \Rightarrow a \sum_{i=1}^{n} x_{i}^{2}+b \sum_{i=1}^{n} x_{i}^{3}+c \sum_{i=1}^{n} x_{i}^{4}=\sum_{i=1}^{n} x_{i}^{2} y_{i}
\end{aligned}
$$


Sendo que o sistema dado pelas equações (4), (5) e (6) pode ser reescrito de forma matricial, pela equação (7), que nos mostra as equações normais para o cálculo do ajuste de curvas quadrático por mínimos quadrados.

$$
\left[\begin{array}{ccc}
n & \sum_{i=1}^{n} x_{i} & \sum_{i=1}^{n} x_{i}^{2} \\
\sum_{i=1}^{n} x_{i} & \sum_{i=1}^{n} x_{i}^{2} & \sum_{i=1}^{n} x_{i}^{3} \\
\sum_{i=1}^{n} x_{i}^{2} & \sum_{i=1}^{n} x_{i}^{3} & \sum_{i=1}^{n} x_{i}^{4}
\end{array}\right] \cdot\left[\begin{array}{l}
a \\
b \\
c
\end{array}\right]=\left[\begin{array}{c}
\sum_{i=1}^{n} y_{i} \\
\sum_{i=1}^{n} x_{i} y_{i} \\
\sum_{i=1}^{n} x_{i}^{2} y_{i}
\end{array}\right]
$$

Em resumo, pelo processo de minimização do cálculo diferencial e integral, devem-se calcular as derivadas parciais da função objetivo em função de $a, b$ e $c$ e igualá-las a zero, formando um sistema linear de ordem $3 \times 3$ descrito na equação (7), oriundo das equações (4), (5) e (6).

\section{RESULTADOS E DISCUSSÃO}

Para calcular a área alagada da barragem do rio Bonito, fez-se a busca no Google maps da região que é objeto de estudo deste artigo. A foto que ilustra a imagem da área que se deseja calcular segue na (figura 4). Na parte inferior do lado direito observa-se a contenção para a barragem. E na parte superior do lado esquerdo tem-se o rio Bonito. Ao seu redor tem-se uma área de proteção ambiental, que segundo dados do presidente da COOIJAM somam um total de 156,3 hectares, entre área alagada e mata. Ainda na parte inferior no lado direito, observa-se a escala, a cada 2,1 centímetros equivale a 200 metros.

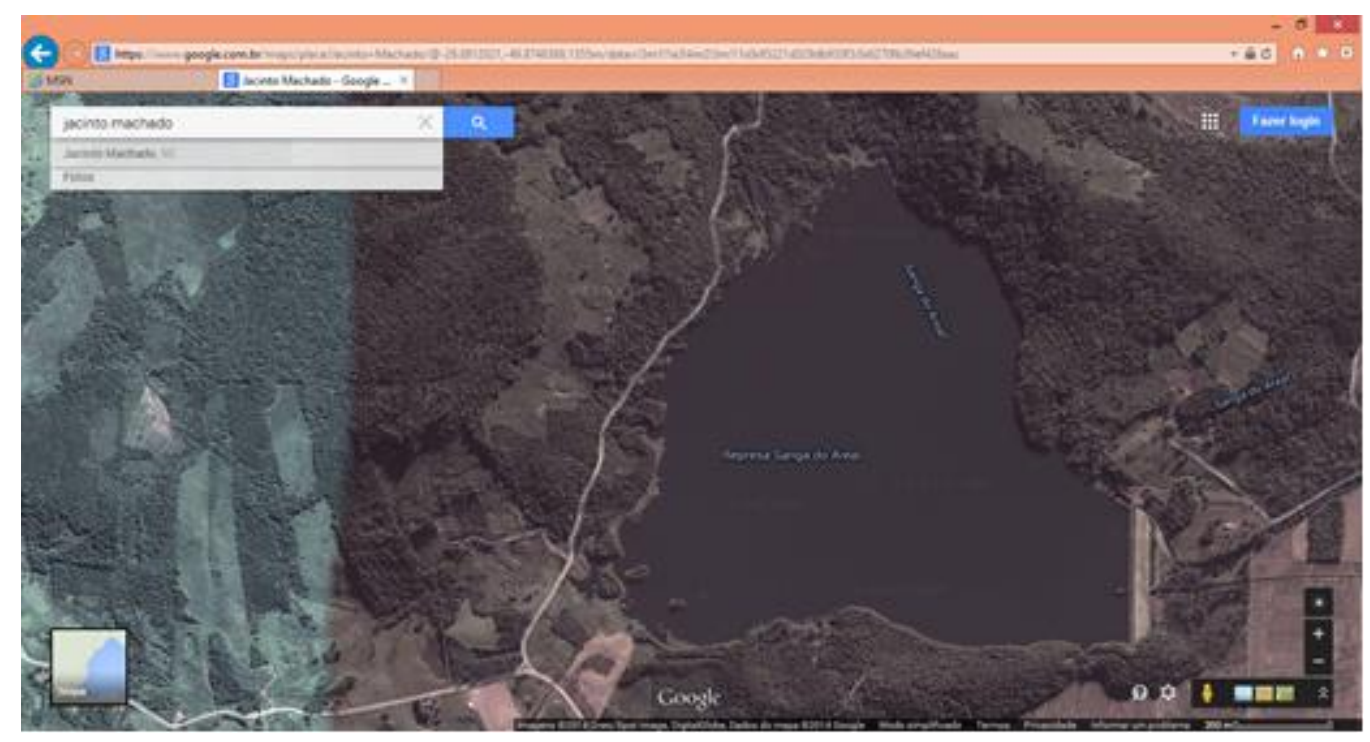

Figura 4 - Mapa da Barragem do Rio Bonito.

Fonte: Google maps, 2014. 
No software geogebra, fez-se a planificação da área a ser calculada (figura 5). Ainda, fezse a coleta dos pontos no plano cartesiano para posterior análise e ajuste utilizando o software graph. Sendo que o objetivo foi encontrar as melhores funções que representam os pontos planificados para o cálculo da área em questão.

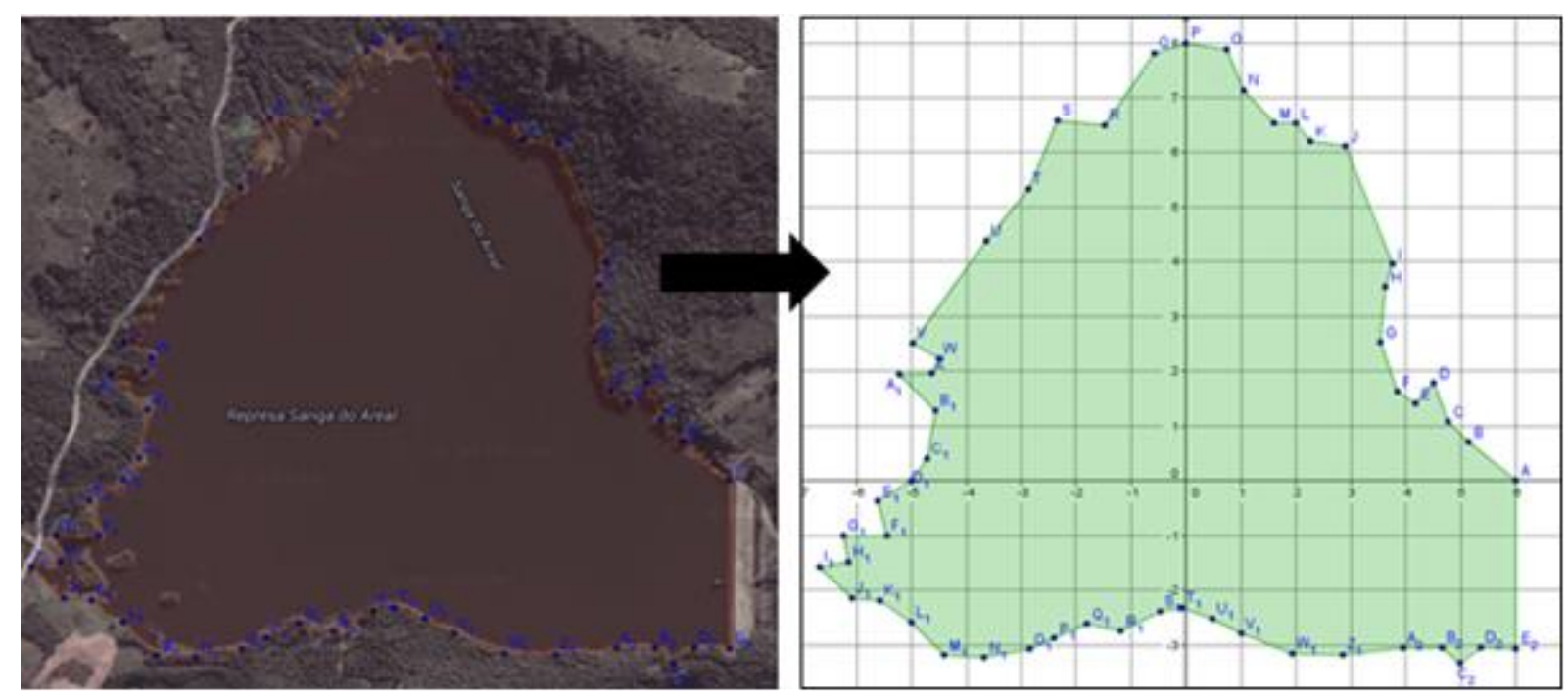

Figura 5 - Planificação da Barragem do Rio Bonito no software geogebra Fonte: Autores, 2014.

A metodologia e a apresentação utilizada no desenvolvimento deste problema, está de acordo com Pires et al (2008).

Segundo Piva (2010), as curvas de contorno, geralmente envolvem coeficientes não exatos, onde a realização dos cálculos manualmente torna-se muito trabalhosa. Porém a utilização do software graph facilita os cálculos.

Neste trabalho, com os pontos apresentados no software geogebra, determinou-se no software graph a linha de tendência que melhor representava o contorno de cada região e consequentemente a função. Então se fez a divisão da área da represa do Rio Bonito em várias sub regiões que são apresentadas nas figuras (6),(9),(10) e (11).

Com os recursos do software graph, foi possível dividir a área representada na figura (6) em 27 subáreas, e para cada região encontrar a melhor linha de tendência, originando a função que representa a área a ser calculada. 


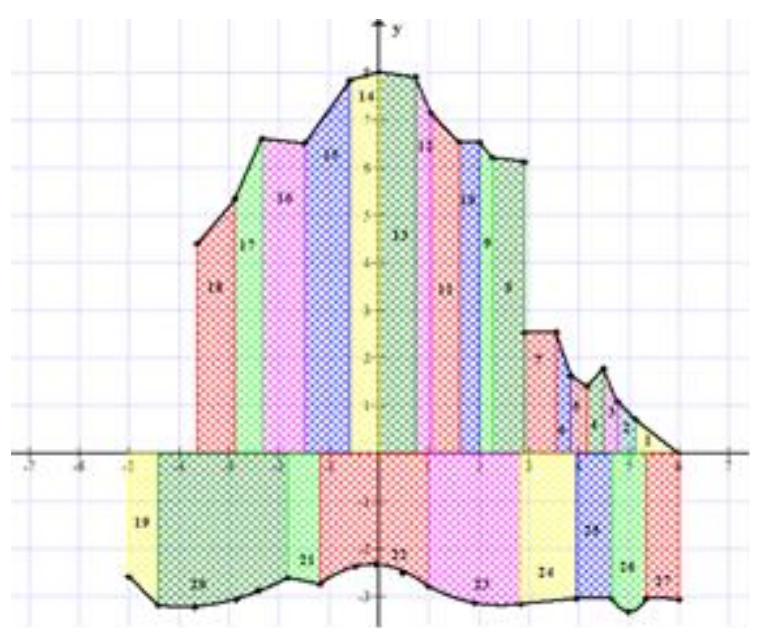

Figura 6 - Áreas calculadas no software graph Fonte: Autores, 2014.

A partir das ferramentas de cálculo do software graph, foi possível calcular através da integral definida o valor de cada região. A tabela (1) apresenta as funções e os intervalos de cada região bem como a área calculada.

Tabela 1- Funções, integral e a área calculada da figura 6.

\begin{tabular}{|c|c|c|c|}
\hline 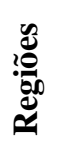 & Funções & Integral & $\begin{array}{l}\text { Resultado } \\
\text { da integral }\end{array}$ \\
\hline 1 & $y=-0,8 x+4,8837$ & $\int_{5,14}^{6}(-0,814 x+4,8837) d x$ & 0,301 \\
\hline 2 & $y=-1,027 x+5,9789$ & $\int_{4,77}^{5,14}(-1,027 x+5,9789) d x$ & 0,3293 \\
\hline 3 & $y=-2,5926 x+13,4467$ & $\int_{4,5}^{4,77}(-2,5926 x+13,4467) d x$ & 0,3841 \\
\hline 4 & $y=1,1515 x-3,4018$ & $\int_{4,17}^{4,5}(1,1515 x-3,4018) d x$ & 0,5247 \\
\hline 5 & $y=-0,697 x+4,3064$ & $\int_{3,84}^{4,17}(-0,697 x+4,3064) d x$ & 0,4999 \\
\hline 6 & $y=-3 x+13,15$ & $\int_{3,54}^{3,84}(-3 x+13,15) d x$ & 0,624 \\
\hline 7 & $y=2,53$ & $\int_{2,9}^{3,54}(2,53) d x$ & 1,6192 \\
\hline
\end{tabular}




\begin{tabular}{|c|c|c|c|}
\hline 8 & $y=-0,125 x+6,4825$ & $\int_{2,26}^{2,9}(-0,125 x+6,4825) d x$ & 3,9424 \\
\hline 9 & $y=-1,2692 x+9,0685$ & $\int_{2}^{2,26}(-1,2692 x+9,0685) d x$ & 1,6549 \\
\hline 10 & $y=6,53$ & $\int_{1,6}^{2}(6,53) d x$ & 2,612 \\
\hline 11 & $y=-1,0893 x+8,2729$ & $\int_{1,04}^{1,6}(-1,0893 x+8,2729) d x$ & 3,8276 \\
\hline 12 & $y=-2,4194 x+9,6561$ & $\int_{0,73}^{1,04}(-2,4194 x+9,6561) d x$ & 2,3296 \\
\hline 13 & $y=-0,1507 x+8$ & $\int_{0}^{0,73}(-0,1507 x+8) d x$ & 5,7999 \\
\hline 14 & $y=0,2881 x+8$ & $\int_{-0,59}^{0}(0,2881 x+8) d x$ & 4,6699 \\
\hline 15 & $y=1,4615 x+8,6923$ & $\int_{-1,5}^{-0,59}(1,4615 x+8,6923) d x$ & 6,5201 \\
\hline 16 & $y=-0,1176 x+6,3235$ & $\int_{-2,35}^{-1,5}(0,1176 x+6,3235) d x$ & 5,5675 \\
\hline 17 & $y=2,3774 x+12,1868$ & $\int_{-2,88}^{-2,35}(2,3774 x+12,1868) d x$ & 3,1641 \\
\hline 18 & $y=1,2338 x+8,8932$ & $\int_{-3,65}^{-2,88}(1,2338 x+8,8932) d x$ & 3,7461 \\
\hline 19 & $y=-1,0508 x-7,8342$ & $\int_{-5}^{-4,41}(-1,0508 x-7,8342) d x$ & 1,7051 \\
\hline 20 & $y=0,1346 x^{2}+1,0661-1,1211$ & $\int_{-4,41}^{-1,81}\left(0,1346 x^{2}+1,0661-1,1211\right) d x$ & 7,9534 \\
\hline 21 & $y=-0,2097 x-2,9995$ & $\int_{-1,81}^{-1,19}(-0,2097 x-2,9995) d x$ & 1,6647 \\
\hline 22 & $y=0,3652 x^{2}-0,1007 x-2,3477$ & $\int_{-1,19}^{1}\left(-0,3652 x^{2}-0,1007 x-2,3477\right) d x$ & 5,4475 \\
\hline
\end{tabular}




\begin{tabular}{l|l|l|l}
\hline 23 & $y=0,1949 x^{2}-0,9497 x-2,0453$ & $\int_{1}^{2,84}\left(0,1949 x^{2}-0,9497 x-2,0453\right) d x$ & 5,6948 \\
\hline 24 & $y=0,1 x-3,454$ & $\int_{1}^{2,84}(0,1 x-3,454) d x$ & 3,4265 \\
\hline 25 & $y=0,0282 x-3,171$ & $\int_{3,94}^{4,65}(0,0282 x-3,171) d x$ & 2,1655 \\
\hline 26 & $y=2,3751 x^{2}-23,751 x+56,046$ & $\int_{4,65}^{5,35}\left(2,3751 x^{2}-23,751 x+56,046\right) d x$ & 2,2638 \\
\hline 27 & $y=0,0462 x-2,7931$ & $\int_{5,35}^{6}(0,0462 x-2,7931) d x$ & 1,9858 \\
\hline
\end{tabular}

Fonte: Autores, (2014)

A soma das áreas calculadas na figura (6) retorna o valor de $80,4234 \mathrm{~cm}^{2}$. Na figura (7) observa-se a área calculada, que é a área sombreada em azul, sendo que a área nesta figura hachurada na cor roxo ainda não foi calculada, pois os autores visualisam uma melhor opção para o seu cálculo, estes cálculos adicionais seguem na sequência, em três novas regiões.

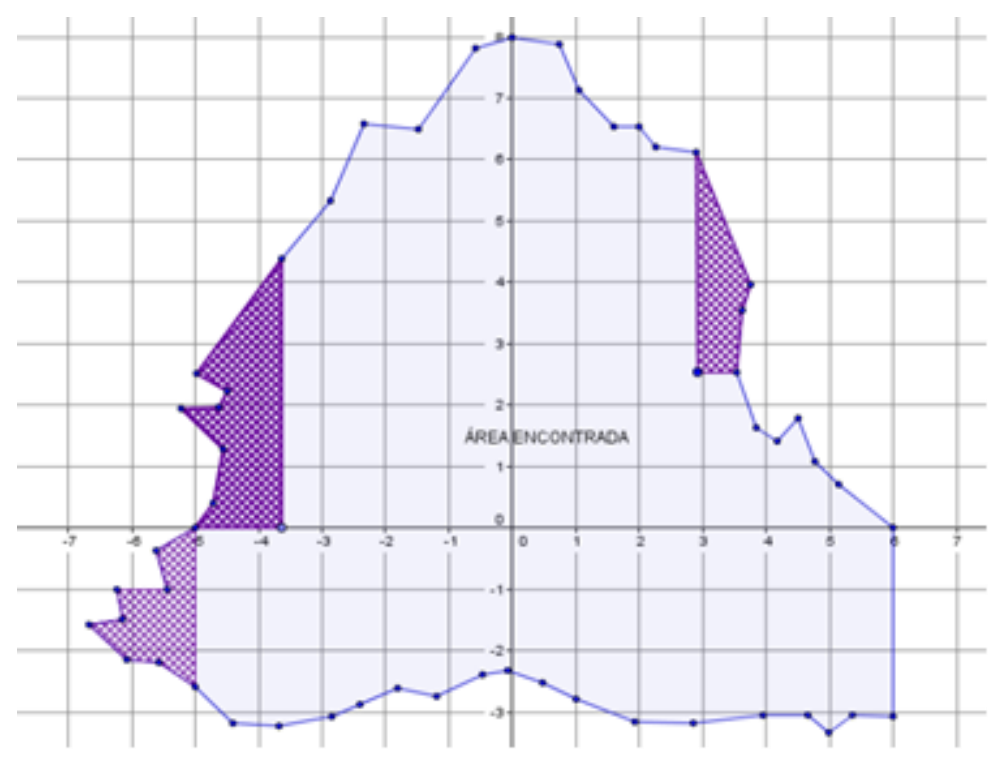

Figura 7 - Área calculada

Fonte: Autores, 2014.

Com o auxilio do software geogebra, utilizando as ferramentas: rotação em torno de um ponto por um ângulo e translação por um vetor construiu-se os novos polígonos das áreas ainda não calculadas. Segundo Medeiros (2012), o software geogebra permite ao usuário fazer sucessivas rotações de uma figura por um ângulo em torno de um ponto central, obtendo figuras congruentes a figura inicial. Fizeram-se essas rotações como mostra a figura (8). 


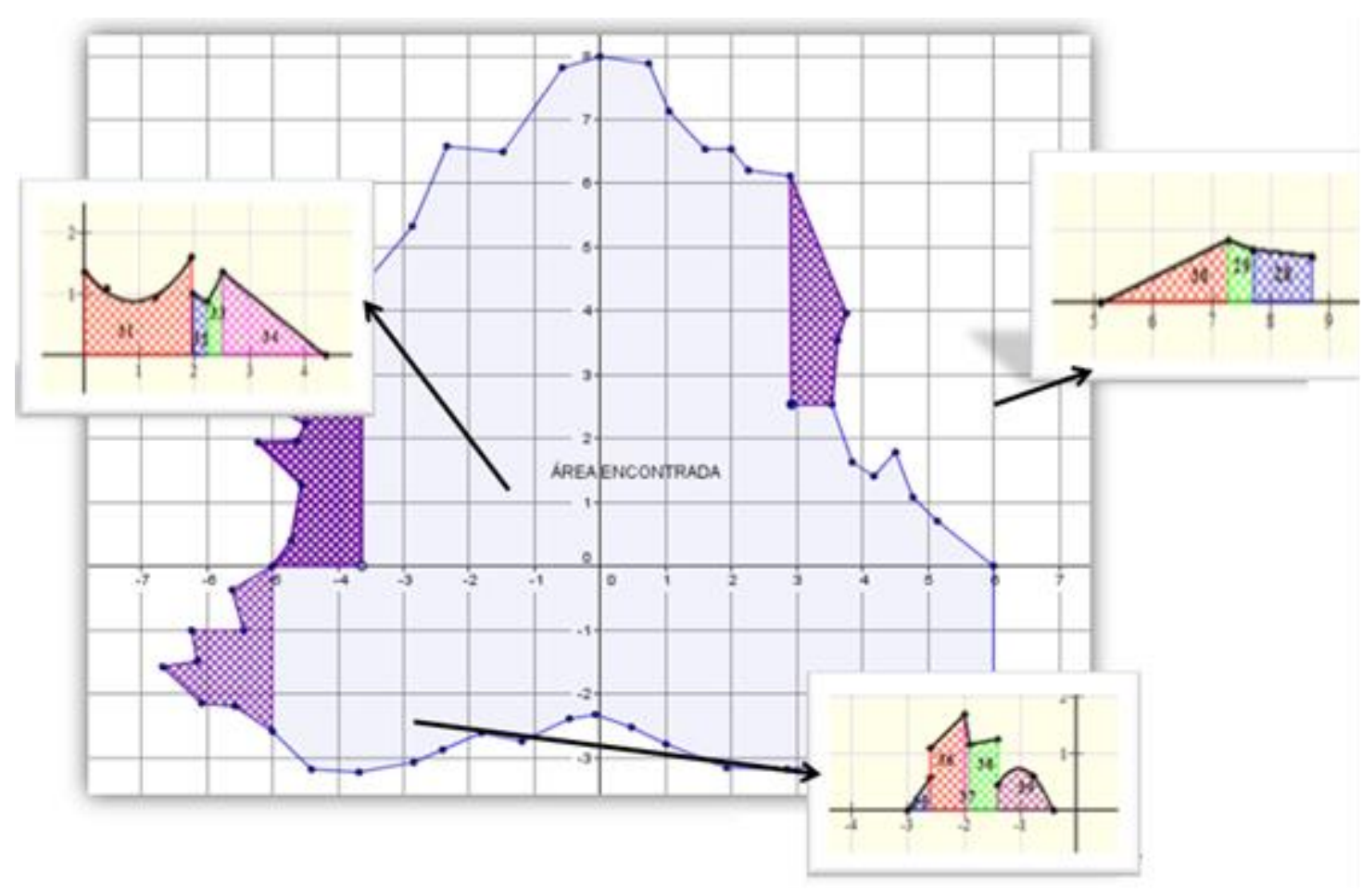

Figura 8 - Rotações

Fonte: Autores, 2014.

Desta forma, novos pontos são apresentados, pois as áreas formam três figuras distintas, representadas nas figuras: (9), (10) e (11). Por fim, no graph foram determinados as funções e o cálculo de suas respectivas áreas.

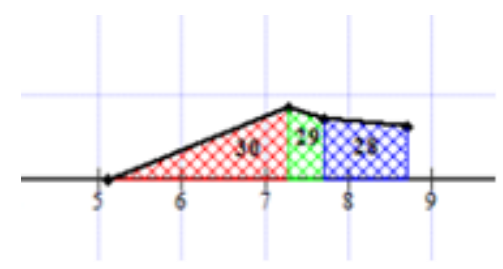

Figura 9 - Áreas calculadas no software graph Fonte: Autores, 2014.

Tabela 2- Funções, integral e a área calculada da figura 9.

\begin{tabular}{|c|c|c|c|}
\hline 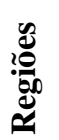 & Funções & Integral & $\begin{array}{l}\text { Resultado da } \\
\text { integral }\end{array}$ \\
\hline 28 & $y=0,3981 x-2,0385$ & $\int_{5,12}^{7,28}(0,3095 x+3,1133) d x$ & 0,9288 \\
\hline
\end{tabular}




\begin{tabular}{l|l|l|l}
\hline 29 & $y=0,3095 x+3,1133$ & $\int_{7,28}^{7,7}(0,3095+3,1133) d x$ & 0,3339 \\
\hline 30 & $y=-0,0891 x+1,4161$ & $\int_{7,7}^{8,71}(-0,0891 x+1,4161) d x$ & 0,6919 \\
\hline
\end{tabular}

Fonte: A autora, 2014

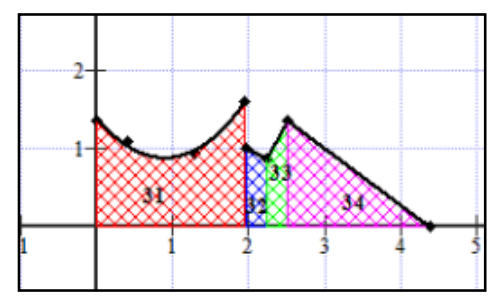

Figura 10 - Áreas calculadas no software graph

Fonte: Autores, 2014.

Tabela 3- Funções, integral e a área calculada da figura 10.

\begin{tabular}{|c|c|c|c|}
\hline 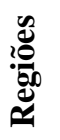 & Funções & Integral & $\begin{array}{l}\text { Resultado da } \\
\text { integral }\end{array}$ \\
\hline 31 & $y=0,645 x^{2}-1,1543 x+1,3977$ & $\int_{0}^{1,94}\left(0,645 x^{2}-1,1543 x+1,3977\right) d x$ & 2,1093 \\
\hline 32 & $y=-0,4815 x+1,9437$ & $\int_{1,94}^{2,23}(-0,4815 x+1,9437) d x$ & 0,2725 \\
\hline 33 & $y=1,75 x-3,0325$ & $\int_{2,23}^{2,51}(1,75 x-3,0325) d x$ & 0,3122 \\
\hline 34 & $y=-0,7273 x+3,1855$ & $\int_{2,51}^{4,38}(-0,7273 x+3,1855) d x$ & 1,2716 \\
\hline
\end{tabular}

Fonte: Autores, 2014

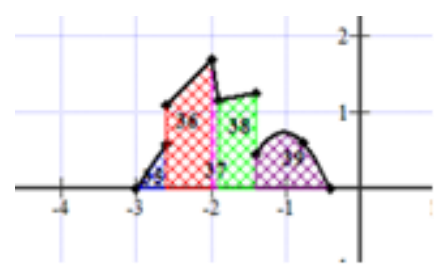

Figura 11: Áreas calculadas no software graph

Fonte: Autores, 2014. 
Tabela 4- Funções, integral e a área calculada da figura 11.

\begin{tabular}{|c|c|c|c|}
\hline 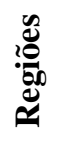 & Funções & Integral & $\begin{array}{l}\text { Resultado da } \\
\text { integral }\end{array}$ \\
\hline 35 & $y=1,439 x+4,3171$ & $\left.\int_{-3}^{-2,59}(1,439 x+4,3171)\right) d x$ & 0,1209 \\
\hline 36 & $y=0,9836 x+3,6375$ & $\int_{-2,59}^{-1,98}(0,9836 x+3,6375) d x$ & 0,8479 \\
\hline 37 & $y=-5,889 x-9,97$ & $\int_{-1,98}^{-1,89}(-5,889 x-9,97) d x$ & 0,1283 \\
\hline 38 & $y=0,1837 x+1,5071$ & $\int_{-1,89}^{-1,4}(0,1837 x+1,5071) d x$ & 0,5905 \\
\hline 39 & $y=-1,9533 x^{2}-4 x-1,3117$ & $\int_{-1,4}^{-0,41}\left(-1,9533 x^{2}-4 x-1,3117\right) d x$ & 0,5436 \\
\hline
\end{tabular}

Fonte: Autores, 2014.

As tabelas (2)-(4) apresentam as funções e os intervalos de cada região dada pelas figuras (9)-(11) bem como a respectiva área calculada. A soma das áreas, representadas nas figuras (6), (9), (10) e (11) retornou o valor de $88.5875 \mathrm{~cm}^{2}$. Assim, o cálculo da área alagada da barragem do rio Bonito segue:

200 metros ….............. 2,10 centímetros

Com base nos dados encontrados e na conversão das unidades tem-se que:

$\left\{\begin{array}{c}40000 \mathrm{~m}^{2} \ldots \ldots . . .4,41 \mathrm{~cm}^{2} \\ \text { Área.....88, } 5875 \mathrm{~cm}^{2}\end{array} \Rightarrow\right.$ Área $=\frac{88,5875 * 40000}{4,41}=803514,7392 \mathrm{~m}^{2}$

A região calculada em hectares tem aproximadamente 80,35 hectares de terra alagada pelas águas do rio Bonito.

\section{CONCLUSÃO}

Neste trabalho foi apresentado o cálculo da área alagada da barragem do rio Bonito, que tem aproximadamente 80,35 hectares usando uma aproximação com o auxílio dos conteúdos do cálculo diferencial e integral, fundamentalmente, integrais definidas e ajuste de curvas além das ferramentas computacionais geogebra e graph.

Dentre as constatações realizadas tem-se que o presente artigo mostrou uma das muitas aplicações do cálculo diferencial e integral. Calcular a área de uma região que não é nada regular 
torna-se um pouco trabalhoso visto que, muitas vezes, nas disciplinas de cálculo o estudante recebe a função a ser integrada, isto é, o processo trabalhado em sala de aula é o contrário deste. Em sala de aula o estudante recebe, em geral, a função, faz o gráfico e calcula sua área. Neste trabalho a área é "conhecida" graficamente, mas as funções que delimitam a área não são conhecidas. Para tanto, fez-se o uso de ajuste de curvas, encontrando assim, uma aproximação para as funções das curvas a serem integradas.

O resultado encontrado é uma boa aproximação da área da região. A COOIJAM (Cooperativa de Irrigação de Jacinto Machado) não sabe o valor exato da área, eles têm uma noção por alto que se equivale ao resultado deste artigo.

Finalmente, o cálculo das funções que representam cada região selecionada da área total a ser calculada, através de ajuste de curvas e do cálculo das integrais definidas, para cada caso, juntamente com os recursos tecnológicos, tornou o trabalho atraente. Acredita-se que este desenvolvimento é de fácil manipulação, visto que seu resultado foi satisfatório, e pode ser aproveitado em sala de aula para apresentar aplicações aos alunos como motivação nas disciplinas de cálculo diferencial e integral e/ou cálculo numérico.

\section{REFERENCIAS}

BASSANEZI, Rodney Carlos. Ensino-aprendizagem com modelagem matemática. São Paulo: Contexto, 2011.

BOYER, Carl B. História da Matemática. São Paulo: Edgar Blücher, 1994.

BRANDÃO, Milena Almeida Leite. DA SILVA, Alessandra Ribeiro. TOGNON, Carlos Henrique. JAFELICE, Rosana Sueli da Mota. O Uso da Modelagem Matemática no Cálculo do Volume de uma maçã. FAMAT em Revista, p. 365383, n.09, out-2007.

KAIBER, Carmen Tereza. RENZ Sandra Pacheco. Cálculo diferencial e integral: uma abordagem utilizando o software maple. PARADIGMA, v.XXIX, p.113-132, n.1, jun-2008.

KOUROPATOV, A; DREYFUS, T. (2009). Integrals as accumulation: a didactical perspective for school mathematics. In: TZEKAKI, M.; KALDRIMIDOU, M.; SAKONIDIS, H. (Eds.). 33 rd Conference of the International Group for the Psychology of Mathematics Education, Proceedings..., v.3, pp, 417-424.

MAGNA ENGENHARIA ltda. Trabalho realizado com colaboração financeira FINEP/BRDE, mar 1982.

Mapa da barragem do Rio Bonito. Disponível em: <https://maps.google.com.br/maps? q=jacinto+ machado+sc \&espv=2\&biw=1024\&bih=653\&dpr=1\&um=1\&ie=UTF8\&sa=X\&ei=KP-UU-qBA8TgsATm64LQCg\&ved=0CAgQ _AUoAQ > Acesso em: 10 jan. 2014

MEDEIROS, Margarete Farias. Geometria dinâmica no ensino de transformações no plano-uma experiência com professores da educação básica. Dissertação de Mestrado- Programa de pós-graduação em Ensino de Matemática/ Mestrado Profissionalizante em Ensino de Matemática. Universidade Federal do Rio Grande do Sul, Porto Alegre, 2012.

PIRES, Andrelizi. NASCIMENTO, Laila Amorim. FABRO, Paloma Nandi. O estudo das integrais: algumas aplicações no contexto. Monografia (Licenciatura em matemática)- UNIVERSIDADE DO SUL DE SANTA CATARINA, Unisul, SC, 2008.

PIVA, Claudia. DORNELES, Lecir Dalabrida. SPILIMBERGO A. Patrícia. Cálculo do Volume de um Sólido de Revolução: Uma Atividade Usando os Softwares Graph e WxMaxima. XXXIII Congresso Nacional de matemática aplicada e computacional, SBMAC, 2010.

SANTOS, Edson Cris Ostomo dos. MOTA, Janine Freitas.BRITO, Alexandre Botelho. FERREIRA, Ronaldo Dias. A utilização do GeoGebra no processo de ensino e aprendizagem da integral: uma articulação entre a pesquisa e a docência. 1 ${ }^{a}$. Conferência Latino Americana de GeoGebra. Anais... ISSN 2237- 9657, pp.129-143, 2012

STEWART, James. Cálculo Vol. 6 a ed. São Paulo: Ed.Cengage Learning, 2011. 